\title{
Antiresorptive medication induced osteonecrosis of the maxilla with facial disfigurement: a case report
}

\author{
Ibrahim AlQuniabut, $\mathrm{MD}^{1} \mathbb{1}$, Hye Jeen Kim, $\mathrm{MD}^{2} \mathbb{D}$, Seong Yong Moon, MD, $\mathrm{PhD}^{3} \mathbb{D}$, Ji Yun Choi, MD, $\mathrm{PhD}^{2} \mathbb{D}$ \\ ${ }^{1}$ Department of Surgery, Unaizah College of Medicine and Medical Sciences, Qassim University, Buraydah, Kingdom of Saudi Arabia \\ ${ }^{2}$ Department of Otolaryngology-Head and Neck Surgery, Chosun University College of Medicine, Gwangju, Rep. of Korea \\ ${ }^{3}$ Department of Oral and Maxillofacial Surgery, School of Dentistry, Chosun University, Gwangju, Rep. of Korea
}

\begin{abstract}
A 77-year-old male presented to our clinic with right cheek swelling, pain, and discharge for 1 month. Oral cavity examination revealed a draining fistula in the hard palate and a tender mass over the right cheek. An enhanced computed tomography scan of the paranasal sinuses showed an enhancing mass with maxillary bone destruction. Fine needle aspiration revealed the presence of pus cells and mucoid material with negative malignant cells. Debridement of loose necrotic tissue resulted in bone necrosis. Therefore, we diagnosed the patient with osteomyelitis induced by antiresorptive medications. A multicourse antibiotic regimen based on culture and sensitivity was initiated, and the patient showed a clinical and radiological response to healing. The patient later developed facial disfiguration due to soft tissue and bone defects with dysfunction of oral competence.
\end{abstract}

Keywords: bisphosphonate; denosumab; facial deformity; maxilla; osteonecrosis

\section{Introduction}

Osteomyelitis is defined as an inflammatory process that affects the bone and its structures [1]. Risk factors leading to osteomyelitis are systemic diseases, such as diabetes mellitus, human immunodeficiency virus, malnutrition, and the use of chemotherapeutic drugs [2]. Facial bone osteomyelitis is rare [1]. Given its long course, outcome uncertainty, and possibility of facial disfigurement because of teeth and bone loss, it is paramount to consider the diagnosis of facial bone osteomyelitis in immunocompromised patients [2].

Osteoporosis is a common disease $[3,4]$ and should be considered a serious public health issue [3]. Studies have focused on finding new treatments for osteoporosis [3]. Generally, they are well-tolerated medications with few side effects, including gastrointestinal symptoms, transit low-grade fever, arthralgia, and bone pain [5].

Recently, bisphosphonate treatment has been reported to have serious adverse effects on bone structure, resulting in osteonecrosis, particularly of the jaw [6]. However, maxillary osteonecrosis remains a rare type, accounting for $25 \%$ of the reported cases of osteonecrosis induced by bisphosphonate [7].

Herein, we report a case of maxillary osteomyelitis induced by bisphosphonate and denosumab (RANK ligand inhibitor) treatment with osteonecrosis of the maxillary bone, resulting in facial disfiguration.

\section{Case report}

A 77-year-old male presented to an otorhinolaryngology 
clinic with right buccal and cheek swelling associated with pain and a purulent discharge from the oral cavity for over 1 month. The patient was referred to a university hospital and diagnosed with a right buccal mass and abscess using contrast-enhanced facial computed tomography (CT). His medical history revealed dementia, myocardial infarction, osteoporosis, and lung cancer, while his surgical history included a right lobectomy due to early-stage lung cancer without adjunctive chemotherapy or radiotherapy. The patient also underwent T11-12, L2-3 fusion with vertebrectomy, and L3-4-5 decompression surgery in 2016 due to a compressive fracture of L1 and L3-5 spinal stenosis. In 2019, he slipped and developed an L4 compression fracture again, but was managed without surgical intervention.

Moreover, his osteoporosis diagnosed in 2014 was managed with oral alendronate, and he received intravenous denosumab along with calcium and vitamin D supplements.

Physical examination of the head and neck revealed a tender right buccal mass associated with a right cervical lymphadenopathy. Endoscopic examination of the nose and paranasal sinuses (PNS) showed a mucoid discharge. Furthermore, oral cavity findings revealed a draining fistula in the hard palate and buccal area (Fig. 1A, B).

A contrast-enhanced CT scan of the PNS showed a premaxilla with surrounding opacification and an enhanced mass in the right cheek associated with destruction of the maxillary bone (Fig. 2). The impression was a hard palate malignancy with right neck metastasis. Therefore, fine needle aspiration was performed on the right cheek mass and was found to be an abscess with mucoid discharge; the results were, negative for tuberculosis and malignant cells. Bacterial culture and sen-
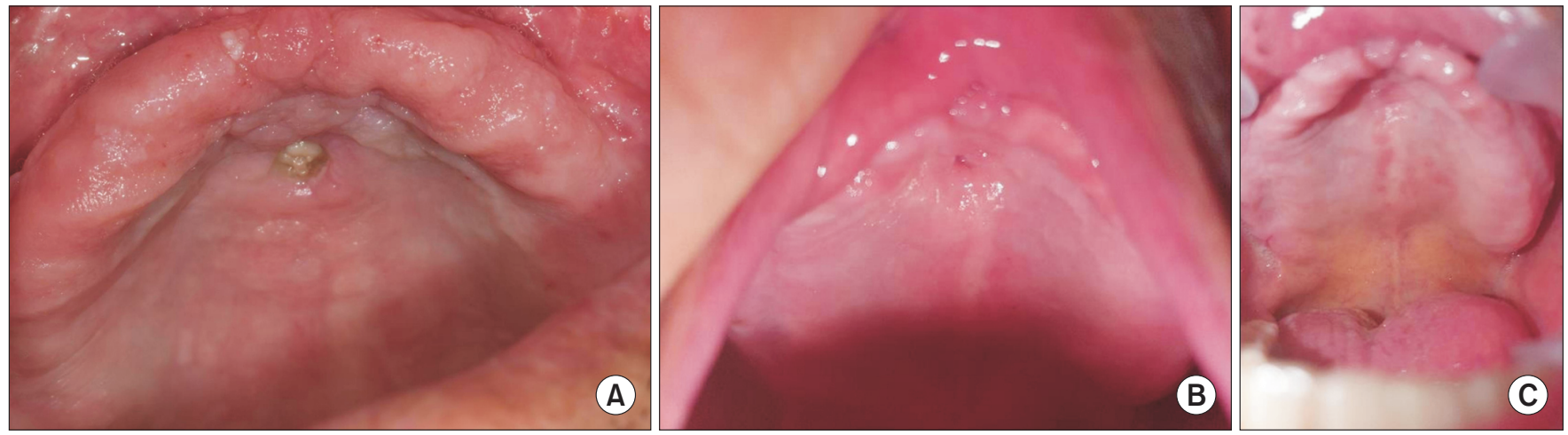

Fig. 1. Oral cavity examination revealed a hard palate draining fistula with purulent discharge (A), during treatment with multicourse antibiotic regimen and micro-debridement of necrotic bone (B), and complete healing of palate fistula after completing the treatment plan (C).
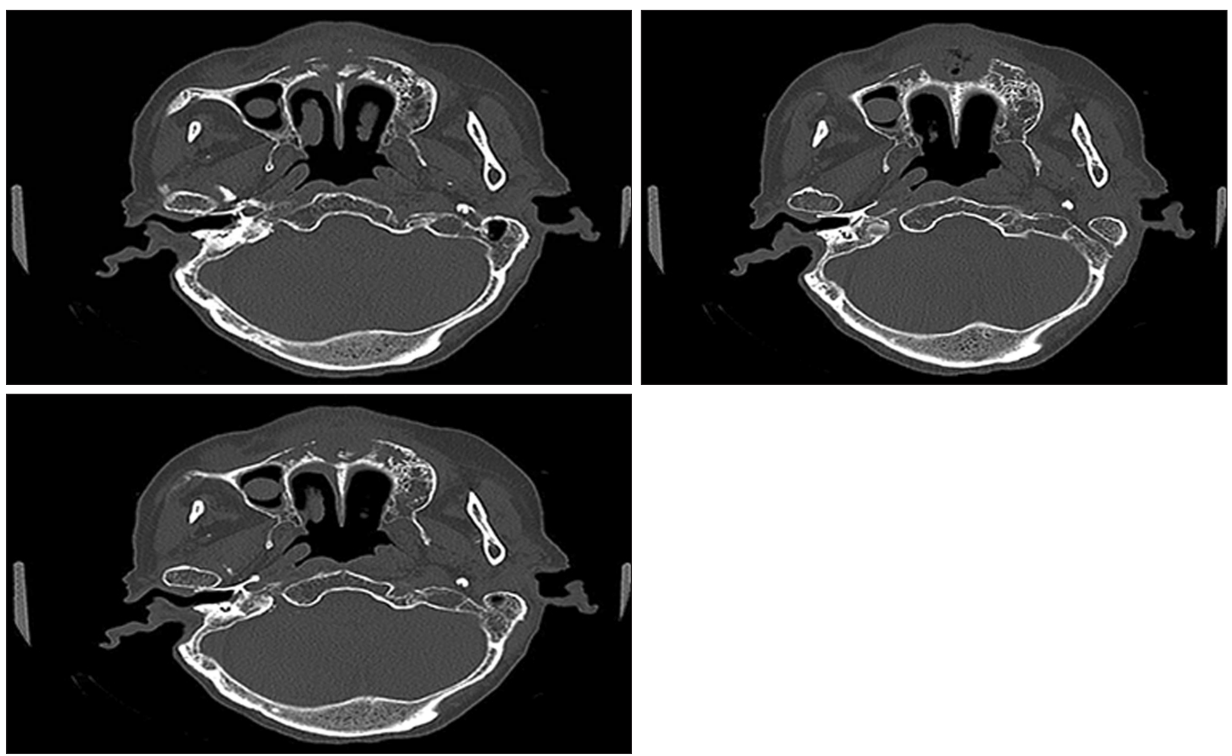

Fig. 2. Axial computed tomography of the paranasal sinuses showed multiple bone sequestrations, irregular bone cortex, and the medullary bone. Destruction of the anterior part of the maxilla gace a motheaten appearance. The air collection at the defect indicated gas forming bacteria (anerobic bacteria). 


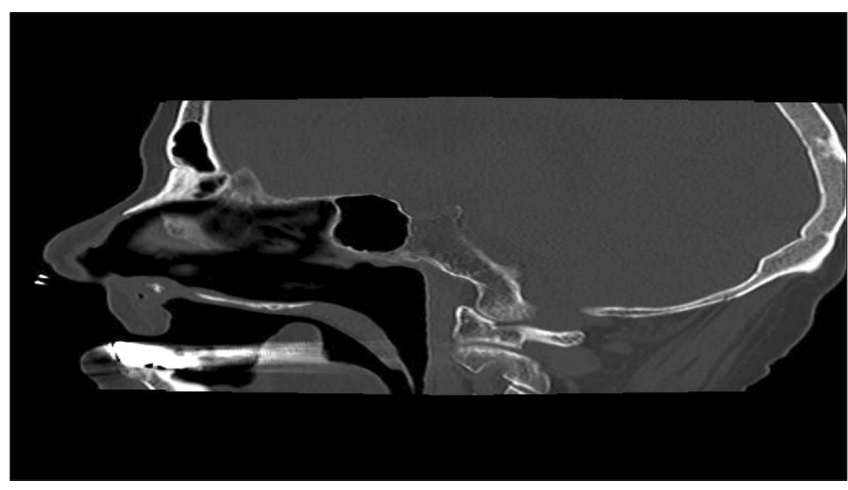

Fig. 3. Post treatment computed tomography of the paranasal sinuses showed the healing fistula on sagittal view and the resolved bone inflammation with stable bony defect of hard palate, which measured $12.07 \mathrm{~mm}$.

sitivity revealed Streptococcus anginosus, which is sensitive to ampicillin, clindamycin, vancomycin, chloramphenicol, and erythromycin. The patient was administered a multicourse oral antibiotic regimen and anti-inflammatory medications, and referred for debridement of the alveolar bone loss, which was performed by a maxillofacial surgeon. Histopathological evaluation revealed several gross fragments of brown soft and hard tissues measuring up to $1.1 \mathrm{~cm} \times 0.7 \mathrm{~cm}$, which were decalcified. Microscopic examination showed inflamed granulation tissue with necrotic bones and bacterial colonization, which was clinically identified as bisphosphonate-induced osteonecrosis. During follow-up, the patient showed significant improvement. His laboratory results were normal, including his white blood cell $\left(7.65 \times 10^{3} / \mu \mathrm{l}\right)$, neutrophil $(79.4 \%)$, and lymphocyte $(11.1 \%)$ counts; C-reactive protein level $(0.17 \mathrm{mg} / \mathrm{dl})$; and erythrocyte sedimentation rate $(15 \mathrm{~mm} / \mathrm{h})$. Furthermore, a follow-up CT scan of the PNS revealed stable destruction of the palate bone with a $12.07 \mathrm{~mm}$ defect (Fig. 3). Later, the patient complained of difficulties controlling suckling liquid food. Thus, we conducted a magnetic resonance imaging scan of the face, which showed no definitive evidence of a residual mass; however, a soft tissue defect on the anterior palate approximately $6.51 \mathrm{~mm}$ in dimension, associated with scar tissue, was noted (Fig. 4).

\section{Discussion}

Osteomyelitis is a dreaded disease due to its long course, uncertain outcomes, and possibility of causing facial disfigurement because of teeth and bone loss resulting in facial scars $[2,6]$, therefore, it is paramount to consider the diagnosis of osteomyelitis of the facial bone in immunocompromised patients. Maxillary osteomyelitis was first described in 1847 [1]. Aside from clas-

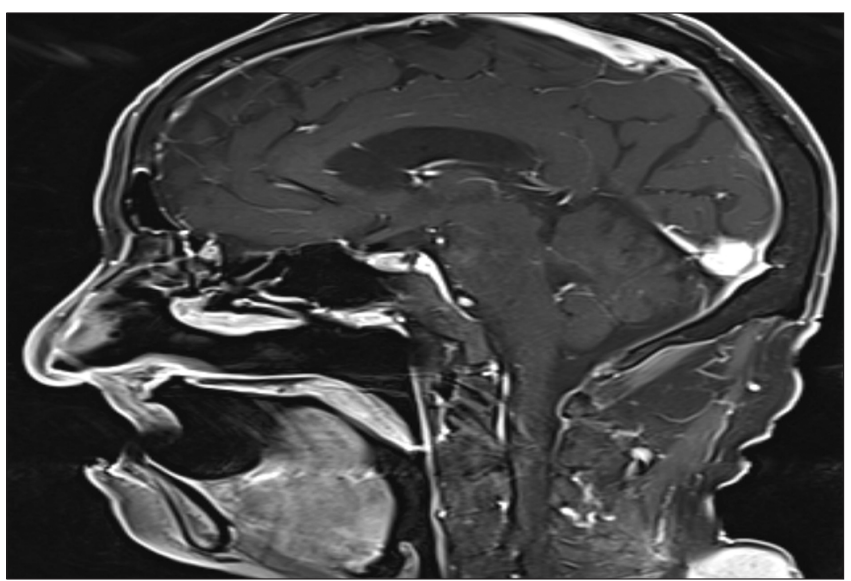

Fig. 4. Magnetic resonance imaging of the paranasal sinuses showed resolved inflammatory process of the maxillary bone and surrounding tissue. Intact soft tissue delineation and continuity with healing of the fistula. The soft tissue defect on sagittal view measured $6.51 \mathrm{~mm}$ resulted in shortening of the anterior-posterior segment of pre maxilla.

sical osteomyelitis, other types of osteomyelitis have emerged as secondary consequences of commonly used drugs [6].

Antiresorptive medications have been used for 30 years for the treating of skeletal disorders, such as bone metastasis and osteoporosis [8]. The main pharmacological effect of these medications is inhibiting bone resorption caused by decreased function of osteoclasts [8]. Thus, cell toxicity induced by this enzymatic inhibition may result in osteonecrosis [6]. Furthermore, the healing process of minor bony lesions is impaired due to the anti-angiogenic effect. Denosumab is a human monoclonal antibody used for its treatment, with bon suppression and turnover equal of greater than that of bisphosphonates. They also differ in their mechanisms of action [7,9].

Recently, patients with exposed facial bones have been referred for the diagnosis of chronic refractory osteomyelitis. The common finding among these cases is the long-term intravenous administration of bisphosphonates [5,8]. Anti-resorptive drug-related osteonecrosis (ARON) was first reported in 2003 and linked to the administration of high doses of bisphosphonates [4]. The pathophysiological mechanism of ARON has been associated with microcirculation disorders and the inhibition of vascular angiogenic growth factor. The maxilla is less prone to osteonecrosis due to abundant vascularization, a thin cortical plate, and a lack of medullary tissue compared to the mandible $[4,6]$.

The American Association of Oral and Maxillofacial Surgeons (AAOMS) defined ARON as constant exposure of necrotic bone in the maxillofacial region for 8 weeks in patients with no his- 
tory of radiotherapy in the neck region, but with a past or active history of bisphosphonate use. The estimated incidence of ARON was between $0.8 \%$ and $12 \%$ [4]. Female (mean age, 66 years) are more commonly affected by ARON due to the management of breast cancer and osteoporosis [5].

Major risk factors for ARON are dental procedures and infection, age $>70$ years, high-dose and long-duration antiresorptive medication use (>36 months), and bone diseases, including cancer. Minor risk factors include organ transplantation, chemotherapy, glucocorticoid use, and diabetes [3,10]. AAOMS has also emphasized that the risk of developing ARON is significantly higher in patients receiving intravenous form [4].

A clear picture may be obtained by a CT scan, which would reveal a peripheral sclerotic rim with diffuse sclerosis, involucrum, central loss of trabecular pattern with internal radiolucent resorptive tracts, and reduction in the alveolar cortex [6]. In more advanced cases, a moth-eaten appearance with poorly defined radiolucency is defined. Moreover, evidence of bone necrosis and marked inflammation are consistent with the histopathological diagnosis of osteonecrosis [2].

The main goal of ARON management is to improve the patients' quality of life, alleviate pain, control infections, and prevent progression of necrosis [5]. Treatment strategies range from conservative protocols for local wounds to aggressive surgical resection of necrotic bones. Conservative protocols, including systemic and oral antibiotics, and debridement of loose necrosis, are effective and may spare patients from complex surgical interventions [5]. Furthermore, the treatment of ARON is difficult, and currently has no definitive management plan [3]. Recently, telopeptide $\mathrm{C}$ type I collagen was reported to be a valuable marker for osteoclast cell activity, which may aid surgical or dental procedures in high-risk patients [4].

Our patient presented with a painful mass on his right cheek, associated with a draining fistula that resembled bisphosphonate-induced osteomyelitis of the jaw, and included swelling of soft tissues, infection, purulent discharge, and bone exposure [8]. However, the presentation was inconsistent with previous studies with regard to the patient's sex, medication use, the location of the disease. Differential diagnoses included alveolar osteitis, chronic sinusitis, gingivitis/periodontitis, sarcoma, chronic sclerosing osteomyelitis, and denosumab-induced osteonecrosis [5]. He was managed with conservative debridement of the necrosis and definitive antimicrobial therapy since his general condition was not indicated for major facial surgery with secondary reconstruction. During follow-up, the patient responded well to our treatment strategy, and the fistula had healed. However, due to defects in the bone and soft tissue induced by necrosis, the patient has a depression in the premaxilla region, which affects oral competence for liquid. Although the classification system widely used for disease prognosis and treatment has been established by Ruggiero et al., the staging system for ARON only has an anatomical consideration, without concern for function or aesthetics $[3,4]$.

\section{Conclusion}

Despite the low incidence of ARON, it is crucial for otolaryngologists to consider a differential diagnosis in patients receiving antiresorptive treatment. Furthermore, optimizing oral health before starting denosumab or bisphosphonate is ideal to avoid such complications. Therefore, educating patients and general practitioners about the rare but devastating side effects of drugs is important to prevent and treat this disease. Finally, a staging system with functional and aesthetic considerations is crucial for guiding management and improving outcomes.

\section{Conflicts of interest}

The authors have nothing to disclose.

\section{References}

1. Arunkumar JS, Naik AS, Prasad KC, Santhosh SG. Role of nasal endoscopy in chronic osteomyelitis of maxilla and zygoma: a case report. Case Rep Med 2011;2011:802964.

2. Habib A, Sivaji N, Ashraf T. Maxillary osteomyelitis: a rare entity. Case Rep Otolaryngol 2016;2016:9723806.

3. Kyrgidis A, Tzellos TG, Toulis K, Antoniades K. The facial skeleton in patients with osteoporosis: a field for disease signs and treatment complications. J Osteoporos 2011;2011:147689.

4. da Silva Santos PS, Oliveira MA, Felix VB. Bisphosphonateinduced maxillofacial osteonecrosis in osteoporotic individuals. Rev Bras Ortop 2015;46:495-9.

5. Santos M, Silveira K, Souza N, Costa D, Inaoka S. Extensive osteonecrosis of the maxilla caused by bisphosphonates: report of a rare case. J Clin Exp Dent 2019;11:e203-7.

6. Guimarães EP, Pedreira FR, Jham BC, de Carli ML, Pereira AA, Hanemann JA. Clinical management of suppurative osteomyelitis, bisphosphonate-related osteonecrosis, and osteoradionecrosis: report of three cases and review of the literature. Case Rep Dent 2013;2013:402096.

7. Smith J, Birkeland AC, McHugh JB, Spector ME. Maxilla os- 
teonecrosis: a differential diagnosis in patients with metastatic cancer on bisphosphonates. J Case Rep Med 2016;5:235969.

8. Kolur T, Nair SC, Kumar B. Osteonecrosis of maxilla secondary to bisphosphonate therapy: a case report. J Maxillofac Oral Surg 2015;14(Suppl 1):52-6.

9. Wimalawansa SJ. Bisphosphonate-associated osteomyelitis of the jaw: guidelines for practicing clinicians. Endocr Pract 2008;14:1150-68.

10. Mosaferi H, Fazlyab M, Sharifi S, Rahimian S. Bisphosphonate-induced osteonecrosis of the maxilla resembling a persistent endodontic lesion. Iran Endod J 2016;11:67-70. 